MATHEMATICS OF COMPUTATION

Volume 79, Number 271, July 2010, Pages 1473-1491

S 0025-5718(09)02306-0

Article electronically published on September 14, 2009

\title{
ERROR BOUND BETWEEN MONOTONE DIFFERENCE SCHEMES AND THEIR MODIFIED EQUATIONS
}

\author{
ZHEN-HUAN TENG
}

\begin{abstract}
It is widely believed that if monotone difference schemes are applied to the linear convection equation with discontinuous initial data, then solutions of the monotone schemes are closer to solutions of their parabolic modified equations than that of the original convection equation. We will confirm the conjecture in this paper. It is well known that solutions of the monotone schemes and their parabolic modified equations approach discontinuous solutions of the linear convection equation at a rate only half in the $L^{1}$-norm. We will prove that the error bound between solutions of the monotone schemes and that of their modified equations is order one in the $L^{1}$-norm. Therefore the conclusion shows that the monotone schemes solve the modified equations more accurately than the original convection equation even if the initial data is discontinuous. As a consequence of the main result, we will show that the half-order rate of convergence for the monotone schemes to the convection equation is the best possible.
\end{abstract}

\section{INTRODUCTION}

In this paper we consider the linear $(p+q+1)$-point monotone difference schemes of the form

$$
\begin{aligned}
& v_{j}^{n+1}=\sum_{s=-p}^{q} a_{s} v_{j+s}^{n}, \\
& v_{j}^{0}=u_{0}(j \Delta x),
\end{aligned}
$$

which are applied to the linear convection equation

$$
\begin{aligned}
& \frac{\partial u}{\partial t}+a \frac{\partial u}{\partial x}=0 \quad \text { for }(x, t) \in \mathbf{R} \times \mathbf{R}^{+}, \\
& u(x, 0)=u_{0}(x) \text { for } \quad x \in \mathbf{R},
\end{aligned}
$$

where $p$ and $q$ are nonnegative integers and $a$ is a constant. The monotone conditions for (1.1) are

$$
a_{s} \geq 0 \quad \text { for } \quad s=-p, \ldots, q
$$

Received by the editor February 2, 2009 and, in revised form, May 20, 2009.

2000 Mathematics Subject Classification. Primary 65M06, 65M15; Secondary 35L45, 35K15.

Key words and phrases. Monotone difference schemes, modified parabolic equation, discontinuous solutions, $L^{1}$-error estimates, linear convection equation.

This work was supported by the National Science Foundation of China (No. 10576001).

(C)2009 American Mathematical Society Reverts to public domain 28 years from publication 
and the consistency conditions are

$$
\sum_{s=-p}^{q} a_{s}=1 \text { and } \sum_{s=-p}^{q} s a_{s}=-\lambda a .
$$

For simplicity of expression in what follows we will only consider the $3(1+1+1)$ point monotone schemes of the form

$$
\begin{aligned}
& v_{j}^{n+1}=\lambda(\gamma-a / 2) v_{j+1}^{n}+(1-2 \gamma \lambda) v_{j}^{n}+\lambda(\gamma+a / 2) v_{j-1}^{n}, \\
& v_{j}^{0}=u_{0}(j \Delta x),
\end{aligned}
$$

but all conclusions obtained in this paper also apply to the $(p+q+1)$-point monotone schemes (1.1). The form (1.4) implies the consistence conditions (1.3), and the arbitrary constant $\gamma$ and Courant number $\lambda=\Delta t / \Delta x$ in the monotone schemes (1.4) satisfy the monotone conditions

$$
\gamma \geq|a| / 2 \text { and } \lambda \leq \frac{1}{2 \gamma}
$$

where $\Delta x$ and $\Delta t$ are space and time steps, respectively. The monotone conditions (1.5) mean that the coefficients of $v_{j-1}^{n}, v_{j}^{n}$ and $v_{j+1}^{n}$ in (1.4) are nonnegative. Here $v_{j}^{n}$ are the numerical solutions, which approximate the exact solution $u(x, t)$ at the point $\left(x_{j}, t_{n}\right)$, where $x_{j}=j \Delta x$ for $j=0, \pm 1, \pm 2, \ldots$ and $t_{n}=n \Delta t$ for $n=0,1,2, \ldots$ are space and time grid points, respectively.

It is known that the monotone schemes are of first-order accuracy and include several popular difference schemes such as the upwind scheme $(\gamma=|a| / 2)$, the Lax-Friedrichs scheme $(\gamma=1 /(2 \lambda))[5$ and the generalized Lax-Friedrichs scheme $(|a| / 2<\gamma<1 /(2 \lambda))$ [7]. Dividing (1.4) by $\Delta t$ the monotone schemes can be written in the form

$$
\frac{v_{j}^{n+1}-v_{j}^{n}}{\Delta t}+a \frac{v_{j+1}^{n}-v_{j-1}^{n}}{2 \Delta x}=\gamma \Delta x \frac{v_{j+1}^{n}-2 v_{j}^{n}+v_{j-1}^{n}}{\Delta x^{2}} .
$$

We will derive modified equations for the monotone difference schemes by using truncation error analysis [2, 3, 10, 12. Let $v(x, t)$ be a smooth function that satisfies the monotone schemes (1.6) at the grid points. Substituting $v(x, t)$ into equation (1.6) and using the Taylor series at $\left(x_{j}, t_{n}\right)$ gives

$$
\left(v_{t}\right)_{j}^{n}+\frac{\Delta t}{2}\left(v_{t t}\right)_{j}^{n}+\mathcal{O}\left(\Delta t^{2}\right)+a\left(v_{x}\right)_{j}^{n}+\mathcal{O}\left(\Delta x^{2}\right)=\gamma \Delta x\left(v_{x x}\right)_{j}^{n}+\mathcal{O}\left(\Delta x^{3}\right) .
$$

Since $\lambda=\Delta t / \Delta x$ is a constant, $\mathcal{O}(\Delta x)$ and $\mathcal{O}(\Delta t)$ are the same order as $\Delta x$ or $\Delta t$ goes to zero. Keeping the first order or second order of $\Delta x$ in the above equation gives

$$
\left(v_{t}\right)_{j}^{n}+a\left(v_{x}\right)_{j}^{n}=\mathcal{O}(\Delta x)
$$

Or

$$
\left(v_{t}\right)_{j}^{n}+\frac{\Delta t}{2}\left(v_{t t}\right)_{j}^{n}+a\left(v_{x}\right)_{j}^{n}=\gamma \Delta x\left(v_{x x}\right)_{j}^{n}+\mathcal{O}\left(\Delta x^{2}\right) .
$$

Differentiating equation (1.7) with respect to $t$ gives

$$
\left(v_{t t}\right)_{j}^{n}+a\left(v_{t x}\right)_{j}^{n}=\mathcal{O}(\Delta x)
$$

and substituting $v_{t}$ from (1.7) into equation (1.9) yields

$$
\left(v_{t t}\right)_{j}^{n}-a^{2}\left(v_{x x}\right)_{j}^{n}=\mathcal{O}(\Delta x) .
$$


By using equation (1.10) to eliminate $v_{t t}$ from equation (1.8) we obtain

$$
\left(v_{t}\right)_{j}^{n}+a\left(v_{x}\right)_{j}^{n}=\Delta x\left(\gamma-\lambda a^{2} / 2\right)\left(v_{x x}\right)_{j}^{n}+\mathcal{O}\left(\Delta x^{2}\right),
$$

where it follows from the monotone conditions (1.5) that

$$
\gamma-\frac{\lambda}{2} a^{2} \geq \frac{|a|}{2}(1-\lambda|a|)>0 .
$$

Remark. Except for a trivial case $(\lambda|a|=1$, a pure translation), the last inequality is a strict inequality.

Truncating the second-order error terms from equation (1.11) gives parabolic equations

$$
\frac{\partial w}{\partial t}+a \frac{\partial w}{\partial x}=\Delta x\left(\gamma-\frac{\lambda}{2} a^{2}\right) \frac{\partial^{2} w}{\partial x^{2}}
$$

which are called parabolic modified equations for the monotone difference schemes. In what follows we write the modified equations as

$$
\frac{\partial w}{\partial t}+a \frac{\partial w}{\partial x}=\varepsilon \frac{\partial^{2} w}{\partial x^{2}}
$$

where $\varepsilon$ is defined by

$$
\varepsilon:=\Delta x\left(\gamma-\frac{\lambda}{2} a^{2}\right)>0
$$

The truncation errors given at the right hand of (1.11) show that if $\lambda<1 /|a|$, then the monotone schemes produce first-order accurate approximations to the convection equation (1.4) and second-order to the modified equations (1.13).

We should point out that the preceding statement is derived by assuming smoothness of the solutions. But it is widely believed that a similar result could be made for discontinuous solutions 2, 1, 6. We will confirm the conjecture in this paper.

It is well known that solutions of the monotone schemes and their parabolic modified equations approach discontinuous solutions of the linear convection equation at a rate only half in the $L^{1}$-norm [4, 9, 13. More precisely, let the initial data $u_{0}(x)$ be a $B V$ function, $v_{\Delta x}(x, t)$ be the numerical solutions of the monotone schemes (1.4) and (1.4) and $u(x, t)$ be the discontinuous solution of (1.2) and (1.2). Then we have

$$
\left\|v_{\Delta x}(\cdot, t)-u(\cdot, t)\right\|_{L^{1}} \leq C\left|u_{0}\right|_{B V}(t \Delta x)^{1 / 2},
$$

where $v_{\Delta x}(x, t)$ is defined by

$$
v_{\Delta x}(x, t)=v_{j}^{n} \quad \text { for } \quad(x, t) \in\left[x_{j}, x_{j+1}\right) \times\left[t_{n}, t_{n+1}\right) .
$$

Similarly, for the modified equations we have the same error estimate

$$
\left\|w_{\Delta x}(\cdot, t)-u(\cdot, t)\right\|_{L^{1}} \leq C\left|u_{0}\right|_{B V}(t \Delta x)^{1 / 2},
$$

where $w_{\Delta x}(\cdot, t)$ is the solution of (1.13) with $w_{\Delta x}(x, 0)=u_{0}(x)$. Here $C$ is a constant, which is independent of $t, x, \Delta t$ and $\Delta x$, but depends on the coefficients of the schemes. The semi-norm $\left|u_{0}\right|_{B V}$ [1] is defined by

$$
\left|u_{0}\right|_{B V}=\sup _{h \neq 0} \frac{1}{|h|}\left\|u_{0}(\cdot+h)-u_{0}(\cdot)\right\|_{L^{1}(\mathbf{R})} .
$$


Remark. We should point out that the half-order rates of convergence given in (1.16) and (1.17) are the best possible 9] and this is also shown in Corollary 1.3 below.

In this paper we will show that the $L^{1}$-error bound between solutions of the monotone schemes (1.4) and that of their modified equations (1.13) is $\mathcal{O}(\Delta x)$. More precisely, we will prove the following theorem.

Theorem 1.1. If $u_{0}(x)$ is a $B V$ function, and $v_{\Delta x}$ and $w_{\Delta x}$ are solutions of (1.4) and (1.13) with initial data $u_{0}(x)$, respectively, then we have

$$
\left\|v_{\Delta x}(\cdot, t)-w_{\Delta x}(\cdot, t)\right\|_{L^{1}} \leq C(\lambda, \gamma, a)\left|u_{0}\right|_{B V} \Delta x,
$$

where $C(\lambda, \gamma, a)$ is a positive constant defined by (4.14).

Remark 1.2. It is noticed that the right-hand side of the estimate given in (1.18) is $C(\lambda, \gamma, a)\left|u_{0}\right|_{B V} \Delta x$, which is, not like (1.16) and (1.17), independent of the time $t$. This means that the estimate is of long time accuracy.

The main theorem implies that the monotone schemes solve the modified equations more accurately than the original convection equation even if the initial data is discontinuous.

As a by-product of the main theorem we can show that the half-order rate of convergence for the monotone schemes to the convection equation given by (1.16) is the best possible. More precisely, we have

Corollary 1.3. Let $u_{0} \in B V, v_{\Delta x}(x, t)$ be the numerical solutions of the monotone schemes (1.4) and (1.4) and $u(x, t)$ be the discontinuous solutions of (1.2) and (1.2). Then for any $t>0$ and $M>0$

$$
\sup _{\left|u_{0}\right|_{B V} \leq M}\left\|v_{\Delta x}(\cdot, t)-u(\cdot, t)\right\|_{L^{1}} \geq \alpha(\gamma, \lambda) M(t \Delta x)^{1 / 2},
$$

provided that $\Delta x$ is small enough. Here $\alpha(\gamma, \lambda)>0$ is a constant depending only on $\gamma$ and $\lambda$.

As another consequence of the main theorem we can show that the monotone schemes approach any other modified parabolic equations at a rate only half in the $L^{1}$-norm. More precisely we have the following corollary.

Corollary 1.4. Let $u_{0}(x) \in B V, v_{\Delta x}$ be solutions of (1.4) and (1.4) and $\bar{w}_{\Delta x}$ be solutions of the following parabolic equations:

$$
\frac{\partial w}{\partial t}+a \frac{\partial w}{\partial x}=\bar{\beta} \Delta x \frac{\partial^{2} w}{\partial x^{2}},
$$

with initial data $u_{0}(x)$, where $\bar{\beta}>0$ is subject to

$$
\bar{\beta} \neq \beta:=\gamma-\frac{\lambda}{2} a^{2} .
$$

Then for any $M>0$ and $t>0$,

$$
\sup _{\left|u_{0}\right|_{B V} \leq M}\left\|v_{\Delta x}(\cdot, t)-\bar{w}_{\Delta x}(\cdot, t)\right\|_{L^{1}} \geq M \frac{|\sqrt{\bar{\beta}}-\sqrt{\beta}|}{\sqrt{\pi}}(t \Delta x)^{1 / 2},
$$

provided that $\Delta x$ is small enough. 
The paper is organized as follows. In section 2 we will prove the main theorem for an important special solution, the Riemann solution, and based on the result we will prove the two corollaries in section 3. In section 4 we will prove the theorem for the general discontinuous solutions. In section 5 we will present some numerical examples to verify the theoretical conclusions. Some comments and discussions are given in the last section.

\section{Riemann initial Data}

The Riemann problem plays an important role in error estimates for discontinuous solutions. In this section we will prove the main theorem for the Riemann initial data.

The linear convection equation (1.2) with piecewise-constant initial data

$$
u_{0}(x)= \begin{cases}u_{-}, & x<0 \\ u_{+}, & x \geq 0\end{cases}
$$

is called the Riemann problem, where $u_{-}$and $u_{+}$are two constants and $u_{0}$ given by (2.1) is called the Riemann initial data. Since the solution of (1.2) and (1.2) can be expressed by

$$
u(x, t)=u_{0}(x-a t),
$$

the Riemann solution $U(x, t)$ of (1.2) and (2.1) is also a piecewise-constant function with a discontinuous line along $x=a t$ :

$$
U(x, t)= \begin{cases}u_{-}, & x-a t<0, \\ u_{+}, & x-a t \geq 0 .\end{cases}
$$

It is known that the solution of the modified equation (1.14) with the initial data $u_{0}(x)$ has the explicit expression

$$
w_{\Delta x}(x, t)=\frac{1}{2 \sqrt{\varepsilon \pi t}} \int_{-\infty}^{\infty} u_{0}(\xi) e^{-\frac{(x-a t-\xi)^{2}}{4 \varepsilon t}} d \xi,
$$

and hence the solution $W_{\Delta x}(x, t)$ of (1.14) with the Riemann initial data (2.1) can be expressed by

$$
W_{\Delta x}(x, t)=u_{-}+\frac{u_{+}-u_{-}}{\sqrt{2 \pi}} \int_{-\infty}^{\frac{x-a t}{\sqrt{2 \varepsilon t}}} e^{-\xi^{2} / 2} d \xi
$$

where $\varepsilon$ is defined by (1.15):

$$
\varepsilon=\Delta x\left(\gamma-\frac{\lambda}{2} a^{2}\right)>0 .
$$

Let $\Phi(x)$ denote the norm distribution function

$$
\Phi(x):=\frac{1}{\sqrt{2 \pi}} \int_{-\infty}^{x} e^{-\xi^{2} / 2} d \xi .
$$

Then the solution $W_{\Delta x}(x, t)$ can be expressed in terms of $\Phi$ :

$$
W_{\Delta x}(x, t)=u_{-}+\left(u_{+}-u_{-}\right) \Phi\left(\frac{x-a t}{\sqrt{2 \varepsilon t}}\right) .
$$

Let

$$
a_{1}=\lambda(\gamma-a / 2), \quad a_{0}=(1-2 \gamma \lambda) \quad \text { and } \quad a_{-1}=\lambda(\gamma+a / 2) \text {. }
$$


Then the monotone schemes can be put in the form

$$
v_{j}^{n+1}=\sum_{l=-1}^{1} a_{l} v_{j+l}^{n},
$$

where $a_{l} \geq 0$ for $l=-1,0,1$ satisfy the consistent conditions:

$$
\sum_{l=-1}^{1} a_{l}=1 \text { and } \sum_{l=-1}^{1} l a_{l}=-\lambda a .
$$

By using (2.8) repeatedly for $n=0, \ldots, n-1$, we find the explicit solution of (1.4) and (1.4):

$$
v_{j}^{n}=\sum_{\substack{n_{-1} \geq 0, n_{0} \geq 0, n_{1} \geq 0 \\ n_{-1}+n_{0}+n_{1}=n}} C_{n}^{n_{-1}, n_{0}, n_{1}} a_{-1}{ }^{n_{-1}} a_{0}{ }^{n_{0}} a_{1}{ }^{n_{1}} u_{0}\left(x_{j-n_{-1}+n_{1}}\right),
$$

where $C_{n}^{n_{-1}, n_{0}, n_{1}}$ is the multinormial coefficient defined by

$$
C_{n}^{n_{-1}, n_{0}, n_{1}}=\frac{n !}{n_{-1} ! n_{0} ! n_{1} !} .
$$

It is easy to know that

$$
\sum_{\substack{n_{-1} \geq 0, n_{0} \geq 0, n_{1} \geq 0 \\ n-1}} C_{n}^{n_{-1}, n_{0}, n_{1}+n_{1}=n} a_{-1}{ }^{n_{-1}} a_{0}{ }^{n_{0}} a_{1}{ }^{n_{1}}=\left(a_{-1}+a_{0}+a_{1}\right)^{n}=1 .
$$

Substituting the Riemann initial data (2.1) into the solution's expression (2.10) and taking account of the identity (2.11) we obtain the solution $V_{j}^{n}$ to the monotone schemes (1.4) with the Riemann initial data (2.1):

$$
V_{j}^{n}=u_{-}+\left(u_{+}-u_{-}\right) \sum_{\substack{j-n_{-1}+n_{1} \geq 0 \\ n_{-1} \geq 0, n_{0} \geq 0, n_{1} \geq 0 \\ n-1+n_{0}+n_{1}=n}} C_{n}^{n_{-1}, n_{0}, n_{1}} a_{-1} n_{-1} a_{0}{ }^{n_{0}} a_{1} n_{1}
$$

or

$$
V_{j}^{n}=u_{-}+\left(u_{+}-u_{-}\right) \sum_{k \leq j} \sum_{\substack{n_{-1}-n_{1}=k \\ n_{-1} \geq 0, n_{0} \geq 0, n_{1} \geq 0 \\ n_{-1}+n_{0}+n_{1}=n}} C_{n}^{n_{-1}, n_{0}, n_{1}} a_{-1}{ }^{n_{-1}} a_{0}{ }^{n_{0}} a_{1}{ }^{n_{1}} .
$$

We will apply the following theorem from probability to prove the main conclusion [8, p. 125].

Theorem 2.1 (Nagaev). Let $X_{1}, \ldots, X_{n}$ be independent identically distributed random variables, $E X_{1}=\mu, E\left(X_{1}-\mu\right)^{2}=\sigma^{2}>0$ and $E\left|X_{1}-\mu\right|^{3}<\infty$. We write

$$
F_{n}(y):=P\left(\frac{S_{n}-n \mu}{\sigma \sqrt{n}}<y\right), \quad S_{n}:=\sum_{l=1}^{n} X_{l}, \quad \varrho:=\frac{E\left|X_{1}-\mu\right|^{3}}{\sigma^{2}} .
$$

Then for all $y \in \mathbf{R}$,

$$
\left|F_{n}(y)-\Phi(y)\right| \leq A \frac{\varrho}{\sqrt{n}(1+|y|)^{3}},
$$

where $A$ denotes a universal positive constant and $\Phi$ denotes the normal distribution function (2.5). 
It is easy to show by induction that if $X_{1}, \ldots, X_{n}$ are independent identically distributed random variables with

$$
P\left(X_{1}=-1\right)=a_{1}, \quad P\left(X_{1}=0\right)=a_{0} \quad \text { and } \quad P\left(X_{1}=1\right)=a_{-1},
$$

then the random variable $S_{n}=X_{1}+\cdots+X_{n}$ has the distribution

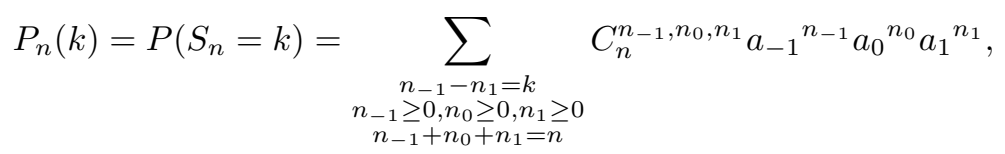

where $k=-n,-n+1, \ldots, n$.

If $a_{-1}, a_{0}, a_{1}$ are defined by (2.7), then

$$
\mu=E X_{1}=\lambda a, \quad \sigma^{2}=E\left(X_{1}-\mu\right)^{2}=2 \lambda\left(\gamma-\frac{\lambda}{2} a^{2}\right),
$$

and

$$
\varrho=\frac{E\left|X_{1}-\mu\right|^{3}}{\sigma^{2}}=\frac{2 \lambda \gamma\left(1-|\lambda a|^{3}\right)-3(\lambda a)^{2}(1-2 \lambda \gamma)+|\lambda a|^{3}(1-|\lambda a|)}{2 \lambda\left(\gamma-\lambda a^{2} / 2\right)} .
$$

Let

$$
y_{k}:=\frac{k-n \mu}{\sigma \sqrt{n}} .
$$

Then

$$
\begin{aligned}
F_{n}(y) & =P\left(\frac{S_{n}-n \mu}{\sigma \sqrt{n}}<y\right)=\sum_{y_{k}<y} P\left(S_{n}=k\right) \\
& =\sum_{y_{k}<y} \sum_{\begin{array}{c}
n_{-1}-n_{1}=k \\
n_{-1} \geq 0, n_{0} \geq 0, n_{1} \geq 0 \\
n_{-1}+n_{0}+n_{1}=n
\end{array}} C_{n}^{n_{-1}, n_{0}, n_{1}} a_{-1}{ }^{n_{-1}} a_{0}{ }^{n_{0}} a_{1}{ }^{n_{1}} .
\end{aligned}
$$

We now turn to the numerical solution $V_{j}^{n}(2.12)$ and write the summing index constraint $k \leq j$ in the form

$$
y_{k}=\frac{k-n \mu}{\sigma \sqrt{n}} \leq \frac{j-n \mu}{\sigma \sqrt{n}},
$$

where $\mu$ and $\sigma$ are defined by (2.16). Some calculations show that

$$
\frac{j-n \mu}{\sigma \sqrt{n}}=\frac{x_{j}-a t_{n}}{\sqrt{2 \varepsilon t_{n}}},
$$

where $x_{j}=j \Delta x, t_{n}=n \Delta t$ and $\varepsilon$ is defined by (1.15). Therefore the inequality (2.19) is equivalent to

$$
y_{k} \leq \frac{x_{j}-a t_{n}}{\sqrt{2 \varepsilon t_{n}}},
$$

and the solution $V_{j}^{n}(2.12)$ can be written as

$$
V_{j}^{n}=u_{-}+\left(u_{+}-u_{-}\right) \sum_{y_{k} \leq \frac{x_{j}-a t_{n}}{\sqrt{2 \varepsilon t_{n}}}} \sum_{\begin{array}{c}
n_{-1}-n_{1}=k \\
n_{-1} \geq 0, n_{0} \geq 0, n_{1} \geq 0 \\
n_{-1}+n_{0}+n_{1}=n
\end{array}} C_{n}^{n_{-1}, n_{0}, n_{1}} a_{-1}{ }^{n_{-1}} a_{0}{ }^{n_{0}} a_{1}{ }^{n_{1}} .
$$

By using (2.18) we can write $V_{j}^{n}$ as

$$
V_{j}^{n}=u_{-}+\left(u_{+}-u_{-}\right) F_{n}\left(\frac{x_{j}-a t_{n}}{\sqrt{2 \varepsilon t_{n}}}\right) .
$$


It follows from (2.20) and (2.6) that

$$
\left|V_{j}^{n}-W_{\Delta x}\left(x_{j}, t_{n}\right)\right|=\left|u_{+}-u_{-}\right|\left|F_{n}\left(\frac{x_{j}-a t_{n}}{\sqrt{2 \varepsilon t_{n}}}\right)-\Phi\left(\frac{x_{j}-a t_{n}}{\sqrt{2 \varepsilon t_{n}}}\right)\right| .
$$

The estimate (2.14) from Theorem 2.1 implies that

$$
\left|V_{j}^{n}-W_{\Delta x}\left(x_{j}, t_{n}\right)\right| \leq\left|u_{+}-u_{-}\right| A \frac{\varrho}{\sqrt{n}\left(1+\left|\frac{x_{j}-a t_{n}}{\sqrt{2 \varepsilon t_{n}}}\right|\right)^{3}} .
$$

Let $V_{\Delta x}(x, t)=V_{j}^{n}$ for $(x, t) \in\left[x_{j}, x_{j+1}\right) \times\left[t_{n}, t_{n+1}\right)$ and

$$
\left\|V_{\Delta x}\left(\cdot, t_{n}\right)-W_{\Delta x}\left(\cdot, t_{n}\right)\right\|_{l^{1}}:=\sum_{j=-\infty}^{\infty}\left|V_{\Delta x}\left(x_{j}, t_{n}\right)-W_{\Delta x}\left(x_{j}, t_{n}\right)\right| \Delta x
$$

Then it follows from (2.21) that

$$
\begin{aligned}
\| V_{\Delta x}\left(\cdot, t_{n}\right) & -W_{\Delta x}\left(\cdot, t_{n}\right) \|_{l^{1}} \leq\left|u_{+}-u_{-}\right| A \sum_{j=-\infty}^{\infty} \frac{\varrho}{\sqrt{n}\left(1+\left|\frac{x_{j}-a t_{n}}{\sqrt{2 \varepsilon t_{n}}}\right|\right)^{3}} \Delta x \\
& \leq 2\left|u_{+}-u_{-}\right| A \frac{\varrho}{\sqrt{n}} \sum_{j=0}^{\infty} \frac{1}{\left(1+\frac{x_{j}}{\sqrt{2 \varepsilon t_{n}}}\right)^{3}} \Delta x \\
& \leq 2\left|u_{+}-u_{-}\right| A \frac{\varrho}{\sqrt{n}}\left(\Delta x+\int_{0}^{\infty} \frac{1}{\left(1+\frac{x}{\sqrt{2 \varepsilon t_{n}}}\right)^{3}} d x\right) \\
& =2\left|u_{+}-u_{-}\right| A \frac{\varrho}{\sqrt{n}}\left(\Delta x+\frac{\sqrt{2 \varepsilon t_{n}}}{2}\right) \\
& =\left|u_{+}-u_{-}\right| A \varrho\left(2+\sqrt{2 \lambda\left(\gamma-\lambda a^{2} / 2\right)}\right) \Delta x,
\end{aligned}
$$

where in the last equality we use the definition (1.15). (2.22) gives the $l^{1}$-error bound.

Now we derive the error bound in the $L^{1}$-norm. Since $\left|W_{\Delta x}\left(\cdot, t_{n}\right)\right|_{B V}=\left|u_{+}-u_{-}\right|$,

$$
\begin{aligned}
&\left\|V_{\Delta x}\left(\cdot, t_{n}\right)-W_{\Delta x}\left(\cdot, t_{n}\right)\right\|_{L^{1}} \\
& \quad \leq \sum_{j=-\infty}^{\infty} \int_{x_{j}}^{x_{j+1}}\left|W_{\Delta x}\left(x_{j}, t_{n}\right)-W_{\Delta x}\left(\xi, t_{n}\right)\right| d \xi+\left\|V_{\Delta x}\left(\cdot, t_{n}\right)-W_{\Delta x}\left(\cdot, t_{n}\right)\right\|_{l^{1}} \\
& \quad \leq\left|W_{\Delta x}\left(\cdot, t_{n}\right)\right|_{B V} \Delta x+\left\|V_{\Delta x}\left(\cdot, t_{n}\right)-W_{\Delta x}\left(\cdot, t_{n}\right)\right\|_{l^{1}} \\
&(2.23)=\left|u_{+}-u_{-}\right| \Delta x+\left\|V_{\Delta x}\left(\cdot, t_{n}\right)-W_{\Delta x}\left(\cdot, t_{n}\right)\right\|_{l^{1}} .
\end{aligned}
$$

Substituting the $l^{1}$-error bound (2.22) into the right-hand side gives

$$
\left\|V_{\Delta x}\left(\cdot, t_{n}\right)-W_{\Delta x}\left(\cdot, t_{n}\right)\right\|_{L^{1}} \leq\left|u_{+}-u_{-}\right|\left[1+A \varrho\left(2+\sqrt{2 \lambda\left(\gamma-\lambda a^{2} / 2\right)}\right)\right] \Delta x
$$


By using the expression (2.17) we obtain

$$
\begin{aligned}
& \left\|V_{\Delta x}\left(\cdot, t_{n}\right)-W_{\Delta x}\left(\cdot, t_{n}\right)\right\|_{L^{1}} \\
& \quad \leq\left|u_{+}-u_{-}\right|\left[1+A \frac{2 \lambda \gamma\left(1-|\lambda a|^{3}\right)-3(\lambda a)^{2}(1-2 \lambda \gamma)+|\lambda a|^{3}(1-|\lambda a|)}{2 \lambda\left(\gamma-\lambda a^{2} / 2\right)}\right. \\
& \left.\quad \times\left(2+\sqrt{2 \lambda\left(\gamma-\lambda a^{2} / 2\right)}\right)\right] \Delta x .
\end{aligned}
$$

Let

$$
\begin{aligned}
C(\lambda, \gamma, a):=1+ & A \frac{2 \lambda \gamma\left(1-|\lambda a|^{3}\right)-3(\lambda a)^{2}(1-2 \lambda \gamma)+|\lambda a|^{3}(1-|\lambda a|)}{2 \lambda\left(\gamma-\lambda a^{2} / 2\right)} \\
& \times\left(2+\sqrt{2 \lambda\left(\gamma-\lambda a^{2} / 2\right)}\right) .
\end{aligned}
$$

Then we obtain

$$
\left\|V_{\Delta x}\left(\cdot, t_{n}\right)-W_{\Delta x}\left(\cdot, t_{n}\right)\right\|_{L^{1}} \leq\left|u_{+}-u_{-}\right| C(\lambda, \gamma, a) \Delta x .
$$

Now we consider the case of $t \neq t_{m}$ for $\forall m \in \mathbb{N}$ and assume that $t=t_{n}+\delta t$ for some $n$, where $0<\delta t<\Delta t$. Therefore

$$
\left\|V_{\Delta x}(\cdot, t)-W_{\Delta x}(\cdot, t)\right\|_{L^{1}}=\left\|V_{\Delta x}\left(\cdot, t_{n}\right)-W_{\Delta x}\left(\cdot, t_{n}+\delta t\right)\right\|_{L^{1}} .
$$

Using the Taylor expansion at $t_{n}$, on account of (1.14), we have

$$
\begin{aligned}
W_{\Delta x}\left(x, t_{n}+\delta t\right) & =W_{\Delta x}\left(x, t_{n}\right)+\delta t \partial_{t} W_{\Delta x}\left(x, t_{n}+\theta \delta t\right) \\
& =W_{\Delta x}\left(x, t_{n}\right)+\delta t\left(-a \partial_{x}+\varepsilon \partial_{x x}\right) W_{\Delta x}\left(x, t_{n}+\theta \delta t\right)
\end{aligned}
$$

where $0<\theta<1$. It follows from (2.4) that

$$
\left\|\left(-a \partial_{x}+\varepsilon \partial_{x x}\right) W_{\Delta x}\left(\cdot, t_{n}+\theta \delta t\right)\right\|_{L^{1}} \leq\left|u_{+}-u_{-}\right|\left(|a|+\sqrt{\frac{2 \gamma-\lambda a^{2}}{2 \pi \lambda}}\right)
$$

and hence

$$
\begin{aligned}
\left\|V_{\Delta x}(\cdot, t)-W_{\Delta x}(\cdot, t)\right\|_{L^{1}} & \leq\left\|V_{\Delta x}\left(\cdot, t_{n}\right)-W_{\Delta x}\left(\cdot, t_{n}\right)\right\|_{L^{1}} \\
& +\delta t\left|u_{+}-u_{-}\right|\left(|a|+\sqrt{\frac{2 \gamma-\lambda a^{2}}{2 \pi \lambda}}\right) .
\end{aligned}
$$

Combining (2.25) and (2.27) gives

$$
\left\|V_{\Delta x}(\cdot, t)-W_{\Delta x}(\cdot, t)\right\|_{L^{1}} \leq\left|u_{+}-u_{-}\right| C(\lambda, \gamma, a) \Delta x
$$

where

$$
\begin{aligned}
C(\lambda, \gamma, a):=1+ & A \frac{2 \lambda \gamma\left(1-|\lambda a|^{3}\right)-3(\lambda a)^{2}(1-2 \lambda \gamma)+|\lambda a|^{3}(1-|\lambda a|)}{2 \lambda\left(\gamma-\lambda a^{2} / 2\right)} \\
& \times\left(2+\sqrt{2 \lambda\left(\gamma-\lambda a^{2} / 2\right)}\right)+\lambda\left(|a|+\sqrt{\frac{2 \gamma-\lambda a^{2}}{2 \pi \lambda}}\right) .
\end{aligned}
$$

We have now completed the proof of Theorem 1.1 for the Riemann initial data. 


\section{LOWER BOUNDS FOR MONOTONE SCHEMES}

In this section we will use the upper bound (2.28) to derive the lower bound estimates (1.19) and (1.21).

We first prove the lower bound (1.19) for the monotone schemes. It follows from (2.2) and (2.3) that for $t>0$,

$$
\begin{aligned}
\left\|W_{\Delta x}(\cdot, t)-U(\cdot, t)\right\|_{L^{1}} & =\left|u_{+}-u_{-}\right|\|\Phi(\cdot)-R(\cdot)\|_{L^{1}} \sqrt{2 \varepsilon t} \\
& =\left|u_{+}-u_{-}\right||| \Phi(\cdot)-R(\cdot) \|_{L^{1}} \sqrt{2 \Delta x\left(\gamma-\frac{\lambda}{2} a^{2}\right) t},
\end{aligned}
$$

where

$$
R(x)= \begin{cases}0, & x<0 \\ 1, & x \geq 0\end{cases}
$$

By using the triangle inequality and the upper bound (2.28), we have

$$
\begin{aligned}
& \left\|V_{\Delta x}(\cdot, t)-U(\cdot, t)\right\|_{L^{1}} \geq\left\|W_{\Delta x}(\cdot, t)-U(\cdot, t)\right\|_{L^{1}}-\left\|V_{\Delta x}(\cdot, t)-W_{\Delta x}(\cdot, t)\right\|_{L^{1}} \\
& \quad \geq\left|u_{+}-u_{-}\right|\|\Phi(\cdot)-R(\cdot)\|_{L^{1}} \sqrt{2 \Delta x\left(\gamma-\frac{\lambda}{2} a^{2}\right) t-\left|u_{+}-u_{-}\right| C(\lambda, \gamma, a) \Delta x} \\
& \quad=\left|u_{+}-u_{-}\right| \sqrt{t \Delta x}\left(\|\Phi(\cdot)-R(\cdot)\|_{L^{1}} \sqrt{2\left(\gamma-\frac{\lambda}{2} a^{2}\right)}-C(\lambda, \gamma, a) \sqrt{\frac{\Delta x}{t}}\right) .
\end{aligned}
$$

Some calculations show that

$$
\|\Phi(\cdot)-R(\cdot)\|_{L^{1}}=\sqrt{\frac{2}{\pi}} .
$$

Therefore

$$
\left\|V_{\Delta x}(\cdot, t)-U(\cdot, t)\right\|_{L^{1}} \geq\left|u_{+}-u_{-}\right| \sqrt{t \Delta x}\left(2 \sqrt{\frac{1}{\pi}\left(\gamma-\frac{\lambda}{2} a^{2}\right)}-C(\lambda, \gamma, a) \sqrt{\frac{\Delta x}{t}}\right) .
$$

This means that if

$$
\Delta x \leq \frac{1}{\pi}\left(\gamma-\frac{\lambda}{2} a^{2}\right) \frac{t}{C(\lambda, \gamma, a)^{2}}
$$

then

$$
\left\|V_{\Delta x}(\cdot, t)-U(\cdot, t)\right\|_{L^{1}} \geq \alpha(\gamma, \lambda)\left|u_{+}-u_{-}\right|(t \Delta x)^{1 / 2},
$$

where

$$
\alpha(\gamma, \lambda)=\sqrt{\frac{1}{\pi}\left(\gamma-\frac{\lambda}{2} a^{2}\right)}>0 .
$$

The inequality (3.2) implies that the lower bound (1.19), with $\alpha(\gamma, \lambda)$ given by (3.3), holds provided $\Delta x$ satisfies (3.1). We have now completed the proof of Corollary 1.3.

Similarly, we can prove the lower bound (1.21) by using (2.28). Let $V_{\Delta x}(\cdot, t)$, $W_{\Delta x}(\cdot, t)$ and $\bar{W}_{\Delta x}(\cdot, t)$ be the solutions of (1.4), (1.13) and (1.20) with the Riemann initial data (2.1), respectively. Then by using the triangle inequality we have (3.4)

$$
\left\|\bar{W}_{\Delta x}(\cdot, t)-V_{\Delta x}(\cdot, t)\right\|_{L^{1}} \geq\left\|\bar{W}_{\Delta x}(\cdot, t)-W_{\Delta x}(\cdot, t)\right\|_{L^{1}}-\left\|W_{\Delta x}(\cdot, t)-V_{\Delta x}(\cdot, t)\right\|_{L^{1}} .
$$


It follows from the expression (2.4) that we have

$$
\bar{W}_{\Delta x}(x, t)-W_{\Delta x}(x, t)=\frac{u_{+}-u_{-}}{\sqrt{2 \pi}} \int_{\frac{x-a t}{\sqrt{2 \beta \Delta x}}}^{\frac{x-a t}{\sqrt{2 \bar{\beta} \Delta x t}}} e^{-\xi^{2} / 2} d \xi,
$$

where

and hence

$$
\beta=\gamma-\frac{\lambda}{2} a^{2} \neq \bar{\beta}
$$

$$
\begin{aligned}
& \left\|\bar{W}_{\Delta x}(\cdot, t)-W_{\Delta x}(\cdot, t)\right\|_{L^{1}} \\
& \quad=\sqrt{2 \beta \Delta x t} \frac{\left|u_{+}-u_{-}\right|}{\sqrt{2 \pi}} \int_{-\infty}^{\infty}\left|\int_{\eta}^{\sqrt{\beta / \bar{\beta}} \eta} e^{-\xi^{2} / 2} d \xi\right| d \eta \\
& \quad=2 \sqrt{\beta \Delta x t} \frac{\left|u_{+}-u_{-}\right|}{\sqrt{\pi}}\left|\int_{0}^{\infty} \eta\left(\sqrt{\beta / \bar{\beta}} e^{-\frac{\beta}{\beta} \eta^{2} / 2}-e^{-\eta^{2} / 2}\right) d \eta\right| \\
& \quad=2 \sqrt{\beta \Delta x t} \frac{\left|u_{+}-u_{-}\right|}{\sqrt{\pi}}|1-\sqrt{\bar{\beta} / \beta}| \\
& =2 \sqrt{\Delta x t} \frac{\left|u_{+}-u_{-}\right|}{\sqrt{\pi}}|\sqrt{\beta}-\sqrt{\bar{\beta}}| .
\end{aligned}
$$

Substituting (3.5) and (2.28) into (3.4) yields

$$
\begin{aligned}
\| \bar{W}_{\Delta x}(\cdot, t) & -V_{\Delta x}(\cdot, t) \|_{L^{1}} \\
& \geq 2 \sqrt{\Delta x t} \frac{\left|u_{+}-u_{-}\right|}{\sqrt{\pi}}|\sqrt{\beta}-\sqrt{\bar{\beta}}|-\left|u_{+}-u_{-}\right| C(\lambda, \gamma, a) \Delta x \\
& =\sqrt{\Delta x t}\left|u_{+}-u_{-}\right||\sqrt{\beta}-\sqrt{\bar{\beta}}|\left(\frac{2}{\sqrt{\pi}}-\frac{C(\lambda, \gamma, a)}{|\sqrt{\bar{\beta}}-\sqrt{\beta}|} \sqrt{\frac{\Delta x}{t}}\right) .
\end{aligned}
$$

The above inequality shows that if

$$
\Delta x \leq \frac{1}{\pi} \frac{(\sqrt{\bar{\beta}}-\sqrt{\beta})^{2}}{C(\lambda, \gamma, a)^{2}} t
$$

then

$$
\left\|\bar{W}_{\Delta x}(\cdot, t)-V_{\Delta x}(\cdot, t)\right\|_{L^{1}} \geq\left|u_{+}-u_{-}\right| \frac{|\sqrt{\bar{\beta}}-\sqrt{\beta}|}{\sqrt{\pi}}(t \Delta x)^{1 / 2} .
$$

The above statement implies Corollaries 1.4. We have now completed the proof of Corollary 1.3 and 1.4 .

\section{Discontinuous initial Data}

In this section we will prove the main theorem 1.1 for the general discontinuous initial data. Assume that $u_{0}(\cdot) \in B V$ and hence the discontinuous solution $u(x, t)=$ $u_{0}(x-a t)$ of (1.2) and (1.2) also belongs to $B V$ space, i.e., $u(\cdot, t) \in B V$. Since $u_{0} \in B V, \lim _{y \rightarrow x-0} u_{0}(y)$ exists for $\forall x \in \mathbf{R}$. For certainty we assume that for $\forall x \in \mathbf{R}$,

$$
u_{0}(x)=\lim _{y \rightarrow x-0} u_{0}(y)
$$


The assumption will make assuming the point-wise initial data given by (1.4) meaningful. Of course, assuming the averaged initial data

$$
v_{j}^{0}=\int_{(j-1 / 2) \Delta x}^{(j+1 / 2) \Delta x} u_{0}(\xi) d \xi
$$

also works. We will estimate the error bound $\left\|v_{\Delta x}(\cdot, t)-w_{\Delta x}(\cdot, t)\right\|_{L^{1}}$ by using the explicit solution's expressions (2.3) and (2.10). Using index substitution $j-n_{-1}+$ $n_{1}=k$ into (2.10) we can write $v_{j}^{n}$ as

$$
v_{j}^{n}=\sum_{k=j-n}^{j+n} \sum_{\substack{n_{-1}-n_{1}=j-k \\ n_{-1} \geq 0, n_{0} \geq 0, n_{1} \geq 0 \\ n-1 \\ n-n_{0}+n_{1}=n}} C_{n}^{n_{-1}, n_{0}, n_{1}} a_{-1}{ }^{n_{-1}} a_{0}{ }^{n_{0}} a_{1}{ }^{n_{1}} u_{0}\left(x_{k}\right)
$$

or

$$
v_{j}^{n}=\sum_{k=-\infty}^{\infty} \sum_{\substack{n_{-1}-n_{1}=j-k \\ n_{-1} \geq 0, n_{0} \geq 0, n_{1} \geq 0 \\ n_{-1}+n_{0}+n_{1}=n}} C_{n}^{n_{-1}, n_{0}, n_{1}} a_{-1}{ }^{n_{-1}} a_{0}{ }^{n_{0}} a_{1}{ }^{n_{1}} u_{0}\left(x_{k}\right),
$$

where $C_{n}^{n_{-1}, n_{0}, n_{1}}=0$ for $n_{-1}-n_{1}<-n$ or $n_{-1}-n_{1}>n$.

We write the solution $w_{\Delta x}\left(x_{j}, t_{n}\right)$ (2.3) into the form

$$
w_{\Delta x}\left(x_{j}, t_{n}\right)=\frac{1}{2 \sqrt{\varepsilon \pi t_{n}}} \int_{-\infty}^{\infty} u_{0}(\xi) e^{-\frac{\left(x_{j}-a t_{n}-\xi\right)^{2}}{4 \varepsilon t_{n}}} d \xi=I+I I,
$$

where $I$ and $I I$ are defined by

$$
\begin{gathered}
I:=\sum_{k=-\infty}^{\infty} \frac{1}{2 \sqrt{\varepsilon \pi t_{n}}} u_{0}\left(x_{k}\right) \int_{x_{k}}^{x_{k+1}} e^{-\frac{\left(x_{j}-a t_{n}-\xi\right)^{2}}{4 \varepsilon t_{n}}} d \xi, \\
I I:=\sum_{k=-\infty}^{\infty} \frac{1}{2 \sqrt{\varepsilon \pi t_{n}}} \int_{x_{k}}^{x_{k+1}}\left(u_{0}(\xi)-u_{0}\left(x_{k}\right)\right) e^{-\frac{\left(x_{j}-a t_{n}-\xi\right)^{2}}{4 \varepsilon t_{n}}} d \xi .
\end{gathered}
$$

We first estimate

$$
\begin{aligned}
& v_{j}^{n}-I=\sum_{k=-\infty}^{\infty}\left(\sum_{\substack{n_{-1}-n_{1}=j-k \\
n_{-1} \geq 0, n_{0} \geq 0, n_{1} \geq 0 \\
n_{-1}+n_{0}+n_{1}=n}} C_{n}^{n_{-1}, n_{0}, n_{1}} a_{-1}{ }^{n_{-1}} a_{0}{ }^{n_{0}} a_{1}{ }^{n_{1}}\right. \\
&\left.\quad-\frac{1}{2 \sqrt{\varepsilon \pi t_{n}}} \int_{x_{k}}^{x_{k+1}} e^{-\frac{\left(x_{j}-a t_{n}-\xi\right)^{2}}{4 \varepsilon t_{n}}} d \xi\right) u_{0}\left(x_{k}\right) .
\end{aligned}
$$

It follows from (2.15) that

$$
P_{n}(j-k)=\sum_{\substack{n_{-1}-n_{1}=j-k \\ n-1 \geq 0, n_{0} \geq 0, n_{1} \geq 0 \\ n-1}} C_{n}^{n_{-1}, n_{0}, n_{1}} a_{-1}{ }^{n_{-1}} a_{0}{ }^{n_{0}} a_{1}{ }^{n_{1}}
$$

and hence

$$
v_{j}^{n}-I=\sum_{k=-\infty}^{\infty}\left(P_{n}(j-k)-\frac{1}{2 \sqrt{\varepsilon \pi t_{n}}} \int_{x_{k}}^{x_{k+1}} e^{-\frac{\left(x_{j}-a t_{n}-\xi\right)^{2}}{4 \varepsilon t_{n}}} d \xi\right) u_{0}\left(x_{k}\right) .
$$


By using the definitions (2.5) and (2.13) we obtain that

$$
\begin{gathered}
\sum_{l=-\infty}^{k-1} \frac{1}{2 \sqrt{\varepsilon \pi t_{n}}} \int_{x_{l}}^{x_{l+1}} e^{-\frac{\left(x_{j}-a t_{n}-\xi\right)^{2}}{4 \varepsilon t_{n}}} d \xi=\frac{1}{2 \sqrt{\varepsilon \pi t_{n}}} \int_{-\infty}^{x_{k}} e^{-\frac{\left(x_{j}-a t_{n}-\xi\right)^{2}}{4 \varepsilon t_{n}}} d \xi \\
=\frac{1}{\sqrt{2 \pi}} \int_{\frac{x_{j}-x_{k}-a t_{n}}{\sqrt{2 \varepsilon t_{n}}}}^{\infty} e^{-\eta^{2} / 2} d \eta=1-\Phi\left(\frac{x_{j}-x_{k}-a t_{n}}{\sqrt{2 \varepsilon t_{n}}}\right)
\end{gathered}
$$

and

$$
\begin{aligned}
& \sum_{l=-\infty}^{k-1} P_{n}(j-l)=\sum_{l=j-k+1}^{\infty} P_{n}(l)=1-\sum_{l=-\infty}^{j-k} P_{n}(l) \\
& =1-\sum_{\substack{y_{l}<\frac{x_{j}-x_{k}-a t_{n}}{\sqrt{2 \varepsilon t_{n}}} \\
P_{n}}} P_{n}(l)=1-F_{n}\left(\frac{x_{j}-x_{k}-a t_{n}}{\sqrt{2 \varepsilon t_{n}}}\right),
\end{aligned}
$$

where

$$
y_{l}=\frac{x_{l}-a t_{n}}{\sqrt{2 \varepsilon t_{n}}} .
$$

Applying summation by parts to (4.3) and using the identities (4.4) and (4.5) gives

$$
\begin{aligned}
v_{j}^{n}-I= & -\lim _{k \rightarrow \infty}\left(F_{n}\left(y_{k-1}\right)-\Phi\left(y_{k-1}\right)\right) u_{0}\left(x_{k}\right)+\lim _{k \rightarrow-\infty}\left(F_{n}\left(y_{k-1}\right)-\Phi\left(y_{k-1}\right)\right) u_{0}\left(x_{k}\right) \\
& +\sum_{k=-\infty}^{\infty}\left(F_{n}\left(\frac{x_{j}-x_{k}-a t_{n}}{\sqrt{2 \varepsilon t_{n}}}\right)-\Phi\left(\frac{x_{j}-x_{k}-a t_{n}}{\sqrt{2 \varepsilon t_{n}}}\right)\right)\left(u_{0}\left(x_{k}\right)-u_{0}\left(x_{k-1}\right)\right) .
\end{aligned}
$$

It follows from (2.5) and (2.18) that

$$
\lim _{k \rightarrow \infty} F_{n}\left(y_{k}\right)=\lim _{k \rightarrow \infty} \Phi\left(y_{k}\right)=1 \text { and } \lim _{k \rightarrow-\infty} F_{n}\left(y_{k}\right)=\lim _{k \rightarrow-\infty} \Phi\left(y_{k}\right)=0 .
$$

Since $u_{0} \in B V$ and the assumption (4.1), $\lim _{k \rightarrow \pm \infty} u_{0}\left(x_{k}\right)$ exist and are finite. Therefore

$$
\lim _{k \rightarrow \pm \infty}\left(F_{n}\left(y_{k-1}\right)-\Phi\left(y_{k-1}\right)\right) u_{0}\left(x_{k}\right)=0
$$

and hence

$$
v_{j}^{n}-I=\sum_{k=-\infty}^{\infty}\left(F_{n}\left(\frac{x_{j}-x_{k}-a t_{n}}{\sqrt{2 \varepsilon t_{n}}}\right)-\Phi\left(\frac{x_{j}-x_{k}-a t_{n}}{\sqrt{2 \varepsilon t_{n}}}\right)\right)\left(u_{0}\left(x_{k}\right)-u_{0}\left(x_{k-1}\right)\right) .
$$

Applying the impotent inequality (2.14) to the right-hand side gives

$$
\left|v_{j}^{n}-I\right| \leq \sum_{k=-\infty}^{\infty} A \frac{\varrho}{\sqrt{n}\left(1+\left|\frac{x_{j}-x_{k}-a t_{n}}{\sqrt{2 \varepsilon t_{n}}}\right|\right)^{3}}\left|u_{0}\left(x_{k}\right)-u_{0}\left(x_{k-1}\right)\right| .
$$

Let

$$
\left\|v_{\Delta x}\left(\cdot, t_{n}\right)-I\right\|_{l^{1}}=\sum_{j=-\infty}^{\infty}\left|v_{j}^{n}-I\right| \Delta x .
$$

Then it follows from (4.6) that

$$
\left\|v_{\Delta x}\left(\cdot, t_{n}\right)-I\right\|_{l^{1}} \leq A \sum_{k=-\infty}^{\infty} \sum_{j=-\infty}^{\infty} \frac{\varrho}{\sqrt{n}\left(1+\left|\frac{x_{j}-x_{k}-a t_{n}}{\sqrt{2 \varepsilon t_{n}}}\right|\right)^{3}} \Delta x\left|u_{0}\left(x_{k}\right)-u_{0}\left(x_{k-1}\right)\right| .
$$


Similar to the estimate (2.22), the second summation term above satisfies

$$
\sum_{j=-\infty}^{\infty} \frac{\varrho}{\sqrt{n}\left(1+\left|\frac{x_{j}-x_{k}-a t_{n}}{\sqrt{2 \varepsilon t_{n}}}\right|\right)^{3}} \Delta x \leq \varrho\left(2+\sqrt{2 \lambda\left(\gamma-\lambda a^{2} / 2\right)}\right) \Delta x
$$

Hence

$$
\begin{aligned}
\left\|v_{\Delta x}\left(\cdot, t_{n}\right)-I\right\|_{l^{1}} & \leq A \varrho\left(2+\sqrt{2 \lambda\left(\gamma-\lambda a^{2} / 2\right)}\right) \Delta x \sum_{k=-\infty}^{\infty}\left|u_{0}\left(x_{k}\right)-u_{0}\left(x_{k-1}\right)\right| \\
& \leq A \varrho\left(2+\sqrt{2 \lambda\left(\gamma-\lambda a^{2} / 2\right)}\right)\left|u_{0}\right|_{B V} \Delta x
\end{aligned}
$$

It is easy to show that

$$
\begin{aligned}
\|I I\|_{l^{1}} & \leq \Delta x \sum_{j=-\infty}^{\infty} \sum_{k=-\infty}^{\infty} \frac{1}{2 \sqrt{\varepsilon \pi t_{n}}} \int_{x_{k}}^{x_{k+1}}\left|u_{0}(\xi)-u_{0}\left(x_{k}\right)\right| e^{-\frac{\left(x_{j}-a t_{n}-\xi\right)^{2}}{4 \varepsilon t_{n}}} d \xi \\
& =\sum_{k=-\infty}^{\infty} \int_{x_{k}}^{x_{k+1}}\left|u_{0}(\xi)-u_{0}\left(x_{k}\right)\right| \frac{1}{2 \sqrt{\varepsilon \pi t_{n}}} \sum_{j=-\infty}^{\infty} e^{-\frac{\left(x_{j}-a t_{n}-\xi\right)^{2}}{4 \varepsilon t_{n}}} \Delta x d \xi
\end{aligned}
$$

We now estimate the second summation term:

$$
\begin{aligned}
& \frac{1}{2 \sqrt{\varepsilon \pi t_{n}}} \sum_{j=-\infty}^{\infty} e^{-\frac{\left(x_{j}-a t_{n}-\xi\right)^{2}}{4 \varepsilon t_{n}}} \Delta x \\
& \leq \frac{1}{2 \sqrt{\varepsilon \pi t_{n}}} \sum_{j=-\infty}^{\infty} \int_{x_{j-1}}^{x_{j}} e^{-\frac{\left(x-a t_{n}-\xi\right)^{2}}{4 \varepsilon t_{n}}} d x \\
& \quad+\frac{1}{2 \sqrt{\varepsilon \pi t_{n}}}\left(e^{-\frac{\left(\left[\left(a t_{n}+\xi\right) / \Delta x\right] \Delta x-a t_{n}-\xi\right)^{2}}{4 \varepsilon t_{n}}}+e^{-\frac{\left(\left(\left[\left(a t_{n}+\xi\right) / \Delta x\right]+1\right) \Delta x-a t_{n}-\xi\right)^{2}}{4 \varepsilon t_{n}}}\right) \frac{\Delta x}{2} \\
& \leq 1+\frac{\Delta x}{2 \sqrt{\varepsilon \pi t_{n}}}=1+\frac{\Delta x}{2 \Delta x \sqrt{\left(\gamma-\frac{\lambda}{2} a^{2}\right) \pi n \lambda}} \\
& \leq 1+\frac{1}{2 \sqrt{\left(\gamma-\frac{\lambda}{2} a^{2}\right) \pi \lambda}},
\end{aligned}
$$

where $[\eta]$ means the largest integer of $\eta$, which is less than or equal to $\eta$. Substituting the above estimation into (4.8) gives

$$
\begin{aligned}
\|I I\|_{l^{1}} & \leq\left(1+\frac{1}{2 \sqrt{\left(\gamma-\frac{\lambda}{2} a^{2}\right) \pi \lambda}}\right) \sum_{k=-\infty}^{\infty} \int_{x_{k}}^{x_{k+1}}\left|u_{0}(\xi)-u_{0}\left(x_{k}\right)\right|, d \xi \\
& \leq\left(1+\frac{1}{2 \sqrt{\left(\gamma-\frac{\lambda}{2} a^{2}\right) \pi \lambda}}\right)\left|u_{0}\right|_{B V} \Delta x
\end{aligned}
$$


It follows from (4.2), (4.7) and (4.9) that

$$
\begin{aligned}
\| v_{\Delta x}\left(\cdot, t_{n}\right) & -w_{\Delta x}\left(\cdot, t_{n}\right)\left\|_{l^{1}} \leq\right\| v_{\Delta x}\left(\cdot, t_{n}\right)-I\left\|_{l^{1}}+\right\| I I \|_{l^{1}} \\
& \leq\left(A \varrho\left(2+\sqrt{2 \lambda\left(\gamma-\lambda a^{2} / 2\right)}\right)+1+\frac{1}{2 \sqrt{\left(\gamma-\frac{\lambda}{2} a^{2}\right) \pi \lambda}}\right)\left|u_{0}\right|_{B V} \Delta x
\end{aligned}
$$

Similar to the estimate (2.23) we have

$$
\left\|v_{\Delta x}\left(\cdot, t_{n}\right)-w_{\Delta x}\left(\cdot, t_{n}\right)\right\|_{L^{1}} \leq\left\|v_{\Delta x}\left(\cdot, t_{n}\right)-w_{\Delta x}\left(\cdot, t_{n}\right)\right\|_{l^{1}}+\left|u_{0}\right|_{B V} \Delta x
$$

and hence we have

$$
\begin{aligned}
\| v_{\Delta x}\left(\cdot, t_{n}\right) & -w_{\Delta x}\left(\cdot, t_{n}\right) \|_{L^{1}} \\
& \leq\left(A \varrho\left(2+\sqrt{2 \lambda\left(\gamma-\lambda a^{2} / 2\right)}\right)+2+\frac{1}{2 \sqrt{\left(\gamma-\frac{\lambda}{2} a^{2}\right) \pi \lambda}}\right)\left|u_{0}\right|_{B V} \Delta x
\end{aligned}
$$

Substituting the $\varrho$ expression (2.17) into the above inequality we obtain

$$
\left\|v_{\Delta x}\left(\cdot, t_{n}\right)-w_{\Delta x}\left(\cdot, t_{n}\right)\right\|_{L^{1}} \leq C(\lambda, \gamma, a)\left|u_{0}\right|_{B V} \Delta x
$$

where

$$
\begin{aligned}
C(\lambda, \gamma, a) & =A \frac{2 \lambda \gamma\left(1-|\lambda a|^{3}\right)-3(\lambda a)^{2}(1-2 \lambda \gamma)+|\lambda a|^{3}(1-|\lambda a|)}{2 \lambda\left(\gamma-\lambda a^{2} / 2\right)} \\
& \times\left(2+\sqrt{2 \lambda\left(\gamma-\lambda a^{2} / 2\right)}\right)+2+\frac{1}{2 \sqrt{\left(\gamma-\frac{\lambda}{2} a^{2}\right) \pi \lambda}}
\end{aligned}
$$

Now we consider the case of $t \neq t_{m}$ for $\forall m \in \mathbb{N}$ and assume that $t=t_{n}+\delta t$ for some $n$, where $0<\delta t<\Delta t$. Similar to the estimate (2.27) we have

$$
\begin{aligned}
\left\|v_{\Delta x}(\cdot, t)-w_{\Delta x}(\cdot, t)\right\|_{L^{1}} & \leq\left\|v_{\Delta x}\left(\cdot, t_{n}\right)-w_{\Delta x}\left(\cdot, t_{n}\right)\right\|_{L^{1}} \\
& +\delta t\left|u_{0}\right|_{B V}\left(|a|+\sqrt{\frac{2 \gamma-\lambda a^{2}}{2 \pi \lambda}}\right) .
\end{aligned}
$$

Combining (4.10) and (4.12) gives

$$
\left\|v_{\Delta x}(\cdot, t)-w_{\Delta x}(\cdot, t)\right\|_{L^{1}} \leq C(\lambda, \gamma, a)\left|u_{0}\right|_{B V} \Delta x
$$

where

$$
\begin{aligned}
C(\lambda, \gamma, a) & =A \frac{2 \lambda \gamma\left(1-|\lambda a|^{3}\right)-3(\lambda a)^{2}(1-2 \lambda \gamma)+|\lambda a|^{3}(1-|\lambda a|)}{2 \lambda\left(\gamma-\lambda a^{2} / 2\right)} \\
& \times\left(2+\sqrt{2 \lambda\left(\gamma-\lambda a^{2} / 2\right)}\right)+2+\frac{1}{2 \sqrt{\left(\gamma-\frac{\lambda}{2} a^{2}\right) \pi \lambda}} \\
& +\lambda\left(|a|+\sqrt{\frac{2 \gamma-\lambda a^{2}}{2 \pi \lambda}}\right) .
\end{aligned}
$$

We have now completed the proof of Theorem 1.1 for the $B V$ initial data. 


\section{Numerical EXPERIMENTS}

In this section we will compute a discontinuous solution of the following linear convection equation by using an upwind monotone difference scheme and show that the $l^{1}$-convergence rate between the upwind solution and its modified solution is one. The linear convection equation is

$$
\begin{cases}\frac{\partial u}{\partial t}+\frac{\partial u}{\partial x}=0 & \text { for } \quad(x, t) \in \mathbf{R} \times \mathbf{R}^{+} \\ u(x, 0)=u_{0}(x) & \text { for } \quad x \in \mathbf{R}\end{cases}
$$

where $u_{0}$ is a Riemann initial data:

$$
u_{0}(x)= \begin{cases}0, & x \leq 0 \\ 1, & x>0 .\end{cases}
$$

The solution of (5.1) and (5.2) is

$$
u(x, t)=u_{0}(x-t),
$$

which has a discontinuous curve $x=t$. The upwind scheme is

$$
\left\{\begin{array}{l}
\frac{v_{j}^{n+1}-v_{j}^{n}}{\Delta t}+\frac{v_{j}^{n}-v_{j-1}^{n}}{\Delta x}=0, \\
v_{j}^{0}=u_{0}\left(x_{j}\right)
\end{array}\right.
$$

where $\Delta x$ and $\Delta t$ satisfy the stable condition:

$$
\lambda=\frac{\Delta t}{\Delta x}<1
$$

and its modified equation is

$$
\left\{\begin{array}{l}
\frac{\partial w}{\partial t}+\frac{\partial w}{\partial x}=\varepsilon \frac{\partial^{2} w}{\partial x^{2}} \\
w(0, x)=u_{0}(x)
\end{array}\right.
$$

where $\varepsilon$ is defined by

$$
\varepsilon:=\frac{1-\lambda}{2} \Delta x>0
$$

It follows from (2.6) that the solution $w_{\Delta x}(x, t)$ of the modified equation (5.5) is

$$
w_{\Delta x}(x, t)=\Phi\left(\frac{x-t}{\sqrt{2 \varepsilon t}}\right) .
$$

In the numerical computation, we set

$$
\lambda=\frac{\Delta t}{\Delta x}=0.5 \quad \text { and } \quad \Delta x=2^{-m} \quad \text { for } \quad m=2,3, \ldots, 8 .
$$

The numerical results are shown in Table1. Here $u(x, t)$, given by (5.3), is the exact solution of the linear convection equation (5.1), $w_{\Delta x}(x, t)$, given by (5.7), is the solution of the modified equation (5.5) and $v_{\Delta x}(x, t)$ is the numerical solution of the upwind difference scheme (5.4). The data given in Table1 1 clearly indicates that the upwind numerical solution archives a first-order rate of convergence in approaching the solution of the modified equation, but a half-order rate in approaching the exact solution of the original convection equation. This verifies the theoretical conclusion of Theorem 1.1 
TABLE $1 . l^{1}$-errors and convergence rates for the numerical solution $v_{\Delta s}$ at $t=1$.

\begin{tabular}{|c|c|c|c|c|}
\hline$t=1$ & \multicolumn{2}{|c|}{$\left\|v_{\Delta x}-w_{\Delta x}\right\|_{l^{1}}$} & \multicolumn{2}{|c|}{$\left\|v_{\Delta x}-u\right\|_{l^{1}}$} \\
\hline$\Delta x$ & $l^{1}$-error & $l^{1}$-rate & $l^{1}$-error & $l^{1}$-rate \\
\hline $2^{-2}$ & 0.1251 & - & 0.3418 & - \\
$2^{-3}$ & 0.0625 & 1.0005 & 0.2209 & 0.6296 \\
$2^{-4}$ & 0.0313 & 1.0001 & 0.1487 & 0.5712 \\
$2^{-5}$ & 0.0156 & 1.0000 & 0.1025 & 0.5374 \\
$2^{-6}$ & 0.0078 & 1.0000 & 0.0715 & 0.5192 \\
$2^{-7}$ & 0.0039 & 1.0000 & 0.0502 & 0.5097 \\
$2^{-8}$ & 0.0020 & 1.0000 & 0.0354 & 0.5049 \\
\hline
\end{tabular}

The numerical solution of the upwind scheme (5.4) with $\Delta x=2^{-3}$, the solution of the modified equation (5.5) and the exact solution of the convection equation (5.1) at $t=1$ are plotted in Figure 1. The figure shows that the numerical solution is much closer to the modified solution than the exact solution.

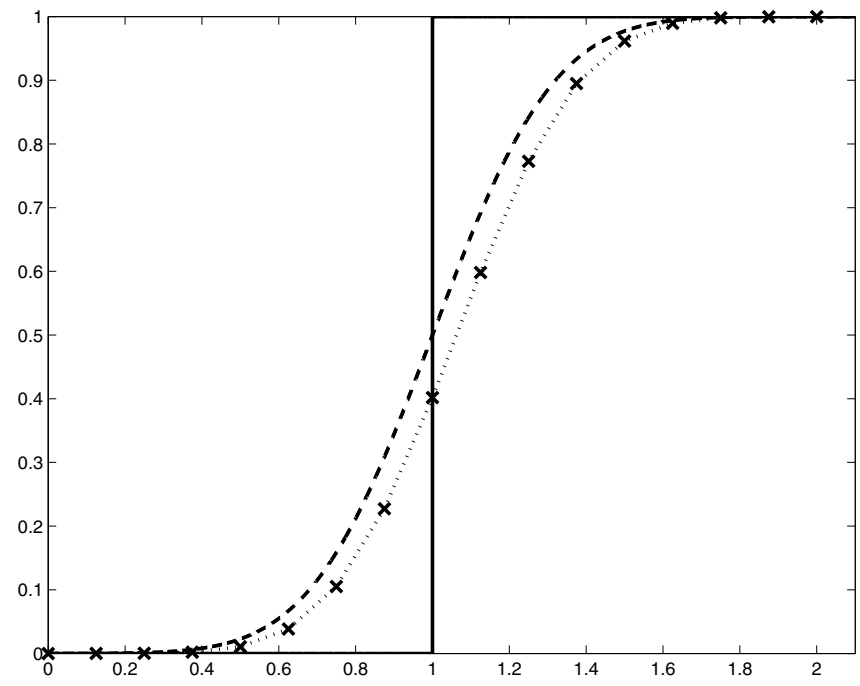

Figure 1. The numerical ".. $\times \ldots$ ", modified " - - " and exact "— solution at $t=1$ with $\Delta x=2^{-3}$.

In order to test the long time accuracy for the numerical solution to approach the modified solution, we compute the numerical solutions by using the upwind scheme (5.4) with $\lambda=1 / 2$ and $\Delta x=2^{-2}, 2^{-3}, \ldots, 2^{-8}$ at $t=1, t=5$ and $t=10$, and the results are shown in Table 2. From the table we see that the $l^{1}$-errors between the numerical solution and the modified solution at different times, i.e., at $t=1$, $t=5$ and $t=10$, are almost the same and the long time accuracy agrees with the theoretical prediction given by Remark 1.2 , 
TABLE 2. Testing the long time accuracy for $\left\|v_{\Delta x}-w_{\Delta x}\right\|_{l^{1}}$ at $t=1, t=5$ and $t=10$

\begin{tabular}{|c|c|c|c|}
\hline \multirow{2}{*}{$\Delta x$} & \multicolumn{3}{|c|}{$\left\|v_{\Delta x}-w_{\Delta x}\right\|_{l^{1}}$} \\
\cline { 2 - 4 } & $t=1$ & $t=5$ & $t=10$ \\
\hline $2^{-2}$ & 0.1251 & 0.1250 & 0.1250 \\
$2^{-3}$ & 0.0625 & 0.0625 & 0.0625 \\
$2^{-4}$ & 0.0313 & 0.0312 & 0.0312 \\
$2^{-5}$ & 0.0156 & 0.0156 & 0.0156 \\
$2^{-6}$ & 0.0078 & 0.0078 & 0.0078 \\
$2^{-7}$ & 0.0039 & 0.0039 & 0.0039 \\
$2^{-8}$ & 0.0020 & 0.0019 & 0.0019 \\
\hline
\end{tabular}

\section{Conclusions}

In this paper we justify that monotone difference schemes give solutions closer to those of the parabolic modified equations than that of the original convection equation and, in particular, prove that the $L^{1}$-error bound between monotone difference schemes and their modified equations is first-order accurate for general $B V$ initial data, which is better than the half-order accuracy between monotone schemes and the convection equation. Furthermore the constant in the error estimate is independent of computational time, and therefore the estimate is of long time accuracy. The results in this paper give a more complete picture for the relationship between the solutions of monotone difference schemes, their parabolic modified equations and the convection equation.

The main conclusion of the paper is for monotone difference schemes approximating the linear convection equation with constant coefficients, but we believe that the result can be extended to the nonlinear conservation laws and this needs some prior estimates for solutions of nonlinear equations. We will report the results elsewhere.

\section{ACKNOWLEDGEMENT}

The author thanks Professor H. Z. Tang of Peking University for many interesting discussions and Professor S. Y. He of Peking University for bringing the author's attention to the important reference [8]. The author also thanks an anonymous referee for valuable comments and suggestions.

\section{REFERENCES}

[1] A. Harten, The artificial compression method for computation of shocks and contact discontinuities. I. Single conservation laws, Comm. Pure Appl. Math. 30 (1977), 611-638. MR0438730 (55:11637)

[2] A. Harten, J. M. Hyman and P. D. Lax, On finite-difference approximation and entropy conditions for shocks, Comm. Pure Appl. Math. 29 (1976), 297-322. MR0413526 (54:1640)

[3] G. Hedstrom, Models of difference schemes for $u_{t}+u_{x}=0$ by partial differential equations, Math. Comp. 29 (1975), 969-977. MR0388797 (52:9631)

[4] N. N. Kuznetsov, Accuracy of some approximate methods for computing the weak solutions of a first-order quasi-linear equation, USSR Comp. Math. and Math. Phys. 16 (1976), 105-119.

[5] P. D. Lax, Weak solutions of nonlinear hyperbolic equations and their numerical computation, Comm. Pure Appl. Math. 7 (1954), 159-193. MR0066040(16:524g) 
[6] R. J. LeVeque, Numerical Methods for Conservation Laws, Birkhäuser Verlag, (1990). MR.1077828 (91j:65142)

[7] J. Liu and Z. Xin, $L^{1}$-stability of stationary discrete shocks, Math. Comp. 60 (1993), 233-244. MR:1159170 (93d:35097)

[8] V. V. Petrov, Sums of Independent Random Variables, Springer-Verlag, (1975). MR0388499 $(52: 9335)$

[9] T. Tang and Z. H. Teng, The sharpness of Kuznetsov's $O(\sqrt{\Delta x}) L^{1}$-error estimate for monotone difference schemes, Math. Comp. 64 (1995), 581-589. MR1270625 (95f:65176)

[10] Z. H. Teng, Modified equation for adaptive monotone difference schemes and its convergent analysis, Math. Comp. 77 (2008), 1453-1465. MR2398776 (2008m:65230)

[11] A. I. Vol'pert and S. I. Hudjaev, Analysis in Classes of Discontinuous Functions and Equations of Mathematical Physics, Martinus Nijhoff Publishers, (1985). MR785938 (86i:00002)

[12] R. Warming and B. Hyett, The modified equation approach to the stability and accuracy analysis of finite-difference methods, J. Comput. Phys. 14 (1974), 159-179. MR0339526 $(49: 4284)$

[13] X. Wen and S. Jin, Convergence of an immersed interface upwind scheme for linear advection equations with piecewise constant coefficients I: $L^{1}$-error estimates, J. Comput. Math. 26 (2008), 1-22. MR 2378582

LMaM \& School of Mathematical Sciences, Peking University, Beijing 100871, China E-mail address: tengzh@math.pku.edu.cn 